\title{
Introspective interviewing for work activities: applying subjective digital ethnography in a nuclear industry case study
}

\author{
Philippe Fauquet-Alekhine ${ }^{1,2,3}$ D $\cdot$ Martin W. Bauer ${ }^{4}$ Saadi Lahlou ${ }^{4}$
}

Received: 15 November 2019 / Accepted: 8 December 2020 / Published online: 5 January 2021

(c) The Author(s) 2021, corrected publication 2021

\begin{abstract}
Subjective Evidence-Based Ethnography (SEBE) is a family of methods developed in digital ethnography for investigation in social science based on subjective audio-video recordings using first-person perspective. Recordings are used for self-confrontation (collect subjective experience, discussion of findings and final interpretation). Several studies applying SEBE methods mentioned "introspection" as a process occurring during self-confrontation and discussed it without providing evidence of its occurrence. This article aimed at clarifying introspection and its occurrence in SEBE. After a literature review addressing introspection, the process of introspection in SEBE was analyzed, depicted and illustrated by a case study. Conditions for introspection to occur in SEBE and the related mechanisms were proposed: it was found that indirect introspection could actually occur but not frequently and could go unnoticed without lessening the quality of the analysis. A refined analysis of introspection during or after the interviews was not identified as an added-value for the activity analysis.
\end{abstract}

Keywords Activity analysis $\cdot$ Cognition $\cdot$ Digital ethnography $\cdot$ Introspection $\cdot$ Memory $\cdot$ Self

\section{Introduction}

Accessing subjects' action during activities inevitably refers to activity analysis and thus to the cognitive task analysis paradigm which regroups methodologies for job or task design and analysis. Two reviews attempted to provide an exhaustive state of the art (Wei and Salvendy 2004; TofelGrehl and Feldon 2013) and a categorization of the methods.

Philippe Fauquet-Alekhine

larsen.sciences@yahoo.fr; p.fauquet-alekhine@1se.ac.uk

Martin W. Bauer

m.bauer@1se.ac.uk

Saadi Lahlou

s.lahlou@1se.ac.uk

1 SEBE-Lab, Department of Psychological and Behavioural Sc., London School of Economics and Political Science, Houghton St., London WC2A 2AE, UK

2 Lab. for Research in Science of Energy, H. Sc., Avoine, France

3 INTRA Robotics Lab, NPP Chinon, BP61, 37420 Avoine, France

4 Department of Psychological and Behavioural Sc., London School of Economics and Political Science, Houghton St., London WC2A 2AE, UK
Among them, process tracing methods have developed with the recent progress of miniaturized camera. Process tracing is capturing expertise during activity performance through audio and/or video recording, followed by an analysis phase of the recordings. When using miniaturized cameras, process tracing methods may be referred to as Subjective EvidenceBased Ethnography (SEBE) as defined by Lahlou (2011). The SEBE is a family of methods developed in digital ethnography for investigation in social science based on subjective audio-video recordings or subfilms (the first-person perspective: Pea 1994; Omodei et al. 2005; Knoblauch et al. 2006; Goldman et al. 2007; Petitmengin 2009; Rix-Lièvre and Lièvre 2010; Lahlou 2011) using miniature video cameras (usually worn at eye-level by subjects: the subcam). Subfilms are then used for self-confrontation with subjects to collect their subjective experience, discussion of findings and final interpretations between researchers and subjects.

Self-confrontation was developed by Von Cranach et al. (1982), and then, on the basis of this work, by Theureau (2002) as a method of investigation of human activity in the framework of his theory of goal-oriented activity. Von Cranach identified three inter-dependent levels of action, each being recoverable by a specific method: (1) the ongoing behavior (acts) are recoverable through audio-visual observation techniques; (2) the cognitive guidance of 
action is recoverable by a self-confrontation of the actor; (3) the organization of social action is recoverable through the confrontation with other actors (social representations). Self-confrontation is a deferred examination of the dynamics of structural coupling subject situation supported jointly by means of reproduction of behavior (e.g., video) and by the researcher as both observer and interlocutor (Theureau 2002). Rieken et al. (2015: 255) applying self-confrontation during digital ethnographic studies for day-work analysis (image-recording of an activity with post-analysis during interviews with subjects) mentioned "introspection" as a process occurring during self-confrontation. Similarly, (Lalhou 2011; Lalhou et al. 2015) and Le Bellu (2011, 2016), also applying digital ethnography for activity analysis, mentioned "introspection" when describing psychological processes during interviews. However, the authors did not characterize the process of introspection and did not provide evidence of its occurrence.

The aim of this article is to clarify two points. First, the notion of introspection is not simple and introspection takes different senses from one research to another; furthermore, "introspection is often viewed with suspicion and seen as an expression of flawed thinking" (Weger et al., 2018: 206): a literature review clarifies this notion in "Literature background". Second, specialists of digital ethnography seem to ask for introspection during the self-confrontation phase of their SEBE-based studies, but it is not clear which forms of introspection occur during these interviews. The research question is thus: does introspection actually occur during SEBE interviews and if yes, in what form? A method for the characterization of introspection during SEBE interviewing is proposed and applied to a case study. Finally, we discuss the added-value of introspection in SEBE.

\section{Literature background}

\subsection{Main streams and controversies}

For Danziger (2015: 702), in a first approach, introspection may simply refer to "the self-observation of mental events". Stated in other words by Vermersch (1994: 203), "the access to knowledge of one's own cognitive functioning may be in a general manner considered as an act of introspection". Written this, any attempt to access and additionally to understand what happens or has happened in a subject's flow of conscious mind 'as experience' is introspection. When understood in this way, psychoanalysis is introspection, meditation, as well as is self-confrontation. Introspection in this sense of supported reflecting on one's life is part of a long tradition of techniques of self-improvement, religious enlightenment and life change or conversion experiences.
We refer to this type of introspection as "macro-introspection", and this will not be our main focus here.

However, for a large part of the scientific community, introspection addresses a more specific psychological process. In his 2006 paper, Overgaard provided an interesting synthesis of what is introspection and how it may be applied. Confronting arguments of renowned pioneers of this concept (Brentano, Comte, James and Wundt), he concluded that 'introspection' is the access to one's inner mental state by oneself (p.630) and "involves an attending to the content of one's consciousness and nothing more than that" for which "the use of an introspective report about the relevant state seems the only possible methodology" (p.631).

Overgaard (2006) pointed to two controversies. A first controversy was that introspection could be considered as an active observation vs a "passive inner perception" so as to avoid "destroying the introspective experience" and thus provide reliable reports (p.630-631). This first controversy involved an all or none argument, with, on the one side, introspectionists like Wundt and Titchener, advocating introspection as 'self-observation' and as the privileged source of psychological data; through strict and laboriously trained methodological procedures, the trained subject would get access to 'pure sensation', purified from all sorts of learned apperceptions. They adhered to the 'constancy hypothesis' between stimulus and sensation, which would later be criticized by the Gestalt psychologist (Koehler 1947: 42ff) to explain perceptual constancy under variables stimulus conditions. Gestalts inquired about phenomenal experience, not introspective accounts. On the other side were Comte, Mills and the positivist tradition, who rejected 'introspection' as an impossibility. Considering 'observation' as the source of data, they rejected 'inner observation' as an illusion because of active attention to mental content, and observation means focused attention, necessarily changing that content.

A second controversy addressed the possibility of accessing inner awareness as retrospective introspection. Overgaard (2006) summarized it so: while Comte tried to demonstrate its impossibility through the argument that a subject cannot split in two parts "so as one part observes the other", James bypassed this problem by turning "introspection" into "retrospection" implying "not to accept introspection of currently conscious states" (p.630). This challenging point led to many studies addressing subjects' verbal reports as valid introspective reports by trying to clarify various conditions of validity. Many of these studies based on the correlation between verbal reports and objective behavioral data related to the same event. To date, the results remain inconclusive. Gaillard et al. (2006), referring to Ericsson and Simon's work (1980), insisted that the main issue was not acceptability (yes or no) overall, but admonished attention to the circumstances of acceptability. Two difficulties needed to be avoided: on the one hand, subjects' concurrent 
verbal reports of their mental states while performing a task interfere with the cognitive process or decrease the speed of performing the activity (Nisbett and Wilson 1977; Ericsson and Simon 1980; Ericsson 2003): if you think about your running you slow down. On the other hand, subjects' retrospective verbal reports lose in reliability due to an inaccurate or partial recall from memory (Gaillard et al. 2006). Many authors advocated combining systematically verbal reports and objective measurements so that to ensure they seized not only what was reported but all that was reportable (Gaillard et al. 2006: 713). In addition, time-delayed retrospection might generate the subjects' interpretation or speculation about their own cognitive processes making it difficult to accept verbal reports as objective and thus acceptable data. In conclusion, the discussion has shifted: rather than dogmatically declaring that all concurrent or retrospective verbal reports are invalid on principle, to make this 'validity' an empirical question in search for circumstances under which verbal reports can usefully be considered. Three considerations come into play: first, the time-lapse between action and verbal report needs to be considered, the longer the more difficult to obtain reliable retrospective reports; second, retrospective reports can be supported by props such as video replay, of which the actor-perspective video seems particularly effective; and thirdly, distinctions needed to be made between 'reports of concomitant awareness' and reports involving increasing mediation including sense making, argumentations and interpretations, inferences and causal attributions or rationalization of actions. Erikson and Simon (1980) developed a memory-based theory of verbalizations to classify reports that are obtained through 'introspection'. We might call this 'micro-introspection'.

Experiments illustrated these empirical efforts such as this of Bechara et al. (1997). The authors studied the subjects' consciousness of self-performance during a simple gambling task (the "Iowa gambling task") by comparing their skin conductance (continuous objective somatic measure) to verbal reports obtained through periodical questioning (subjective data). The task consisted in choosing a card among four decks and maximizing gains on a series of trials, a card resulting in a win or a loss when playing for money. Unknown to the participants, some decks were advantageous, as opposed to others. They found out that subjects started selecting cards from advantageous decks and skin conductance increased just before selecting a disadvantageous card before the subjects were able to verbally explain their choice. For Bechara et al., this situation of dissociation between performance and verbal reports could be interpreted as evidence of unconscious knowledge accessed through introspective verbal reports and confirmed by objective data. A replicated experiment by Maia and McClelland (2004) used a more elaborate questionnaire. They found out that the dissociation vanished and they concluded that there was no evidence of unconscious knowledge and thus no introspective. However, Overgaard (2006) highlighted that the original experiment complied with the introspection need to put the subjects in a "passive inner perception" using simple questions. On the contrary, using an elaborate questionnaire, researchers of the replicated experiment implemented an active observation of mental states and destroyed the introspective experience. Simple questions induced simple answers conversely to elaborate questionnaires. This echoes Titchener's viewpoint (as reported by Danziger 2015), who was "a major exponent of experimental introspection", and who "demanded that introspective descriptions should be in terms of simple, irreducible units and should abstract from any meaning that the stimulus might have" (p.703), since "additional cognitive activity must necessarily change the sequence of mediating thoughts" (Ericsson 2003: 11). Ericsson undertook an analysis regarding factors decreasing validity of verbal reports: "The first arises when the investigators try to obtain more information than the subjects' thought sequences can provide. [...] Second, investigators often ask the subjects to describe their methods for solving problems at the end of the experiment, when they have completed a long series of different tasks. If the subjects generated and consistently applied a general strategy for solving all of the problems, they should be able to respond to such requests easily with a single memory retrieval. But the subjects typically employ many methods and shortcuts and even change their strategies during the experiment, through learning. Under such circumstances, the subjects would have great difficulty describing a single strategy used consistently throughout the experiment, even in the unlikely event that they were motivated and able to recall most of the relevant thought sequences. It is, therefore, not surprising that the subjects' descriptions are imperfectly related to their averaged performance during the entire experiment" (Ericsson 2003: 15). This also led to the assumption that analysts may have an introspective approach with the aim of obtaining introspective verbal reports from subjects about whom they intend to access the mental state but they may be unable to create favorable conditions for introspection. According to Gaillard et al. (2006: 714), "authors widely agree on the validity and on the reliability of verbal data as a source of information about cognitive processes as long as they are elicited with care and interpreted with full understanding of the circumstances under which they were obtained". Introspection is rehabilitated from Positivist denial and rescued from Wundtian dead ends; Brentano (1874: 42ff) showed the middle way of careful methodological procedures (e.g., support for retrospection of awareness, time-lapse considered) and data analysis considering different types 
of verbal production; not all reports obtained are 'introspective', many involve communicative reasoning. Thus introspection also 'democratised' in the sense that everybody is capable of doing so; for Wundt and Titchener, only researchers trained for hundreds of hours and had acquired the 'right attitude' were allowed to 'introspect' (Schwitzgebel 2004).

\subsection{Characterizing introspection from verbal characteristics}

Gaillard et al. (2006), after reviewing and discussing results regarding the contribution of introspection to implicit learning, suggested three criteria to validate the acceptability of verbal reports as introspective:

- Instructions given to subjects for verbalization in either a general or a specific manner must not have any effect on the introspective cognitive processes (limiting the subject's answers to a few possible categories such as "yes" or " "no" or, on the contrary, forcing subjects to produce elaborated and exhaustive description might limit or spoil the introspection process),

- Verbal reports must be complete, consisting of all the information the subjects have about their own cognitive processes,

- Verbal reports must be consistent with other empirical data on behavior.

The last point should be considered as a bilateral relationship: if empirical data on behaviour leads to validating verbal reports, similarly verbal reports lead to explaining empirical data. This is well illustrated by the "Iowa gambling task" experiment reported above: verbal reports were corroborated by the existence of variations of skin conductance and variations of skin conductance were explained by verbal reports; without verbal reports, researchers would have perhaps never known that variations of skin conductance were related to an unconscious knowledge of advantageous decks.

When this criterion is not respected, Gaillard et al. (2006: 713) use the expression "retrospective verbal reports" (shared by other researchers) potentially suffering from a bias due to the fact that "subjects may forget or inaccurately recall the relevant features of the experimental situation, which of course cannot be interpreted as implying that they were unaware when engaged in the task." For these retrospective verbal reports to be considered similar to introspective verbal reports, Ericsson (2003: 13), on the basis of a prior review, suggested that the time to generate these reports had to occur less than $5 \mathrm{~s}$ after the event described so that "the participants can recall their sequence of thoughts reasonably accurately". However, Gaillard did not include this time criterion perhaps, because it characterizes more the way verbal reports were obtained than their validity.

\subsection{Characterizing introspection from memory aspects}

As we can see, applying introspection during work activities implies concurrent verbal reports. Authors qualify the investigation as retrospection or indirect introspection as proposed by (Titchener 1912 and Kriegel 2013) when performed during interviews in an attempt to access mental states (see also Piccinini 2003: concurrent introspective reports and retrospective introspective reports). Nevertheless, implementation does not seem easy whether it be for introspection or retrospection.

As recalled by Kriegel (2013: 1172), direct introspection occurs simultaneously with the introspected event, whereas indirect introspection occurs later and involves recollection of past events; therefore, in terms of memory (Fig. 1), the former relates to short term memory (or working memory) when the latter relies on episodic memory (one of the forms of explicit memory) or procedural memory (one of the forms of implicit memory) used to recall past events (Piccinini 2003).

- Sensory Memory refers to the ability to retain sensory information through the five senses.

- Short Term Memory refers to the capacity to keep available information for a short period of time.

- Long Term Memory refers to the capacity to hold a large amount of information for a long period.

- Encoding process: the perceived item of interest is converted into a construct that can be stored, and then recalled later from Short Term Memory to Long Term Memory)

- Storage process: retaining information in either of the three-stage memory, but mostly in Long Term Memory

- Consolidation process: stabilizing a memory trace after initial acquisition

- Retrieval process: re-accessing events or information from the past previously encoded and stored

- Implicit Memory refers to memories storing previous experiences to perform of a task without conscious awareness of these (including Priming Memory, Procedural Memory and (non) Associative Learning Memory).

- Explicit Memory refers to memories that can be consciously recalled.

- Episodic Memory contains past personal experiences (e.g., time, places, and related emotions).

- Semantic Memory refers to meanings, understandings and other concept-based knowledge. 


\section{MODAL MODEL OF MEMORY}

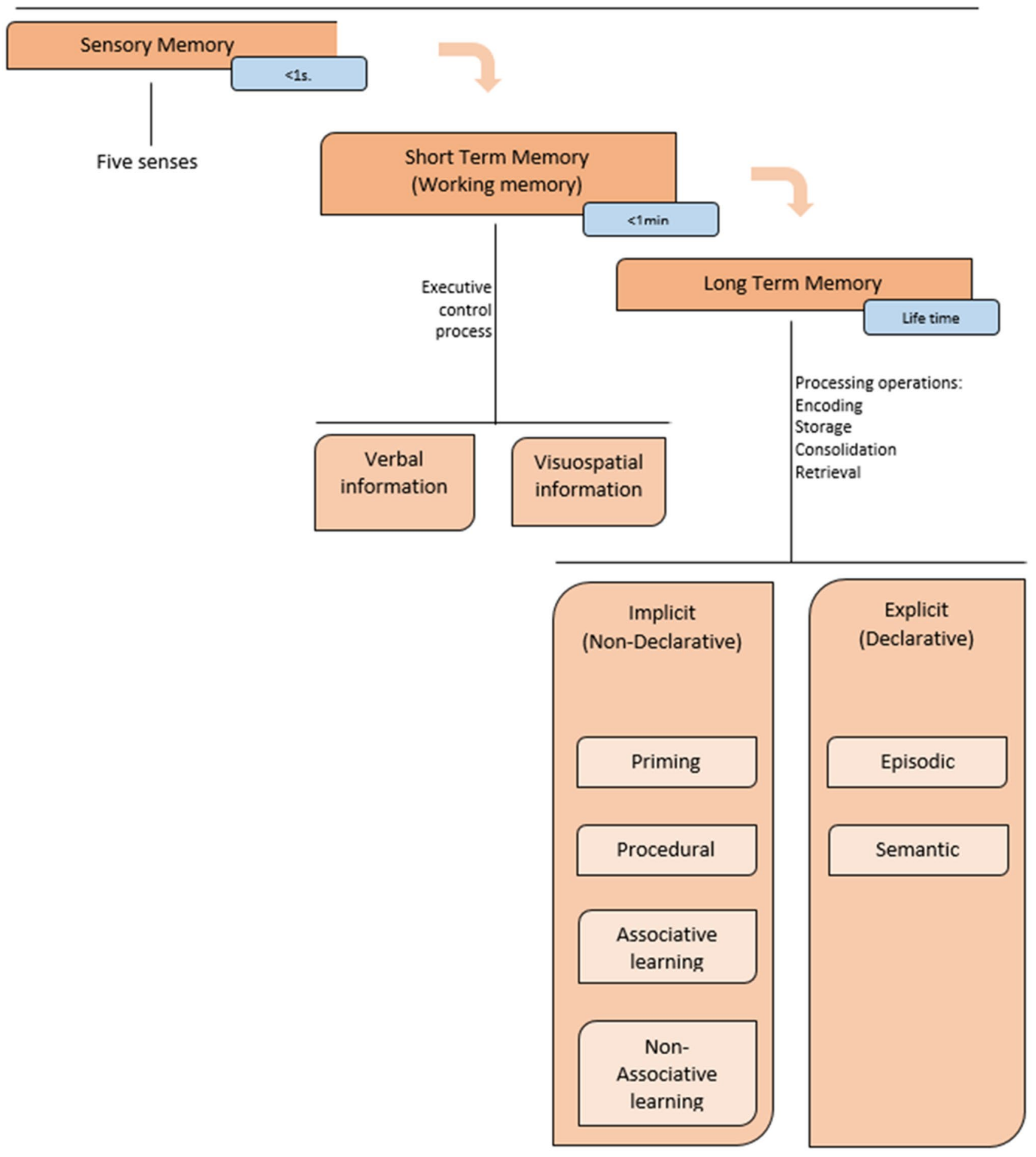

Fig. 1 Multi-store or modal model of memory (adapted from Atkinson-Shiffrin 1968) is the model generally adopted by the scientific community (Velez-Pardo and Jimenez-Del-Rio 2015). Memory is a three-stage sequence: Sensory Memory, Short Term Memory and Long Term Memory 


\subsection{Introspection and the SEBE}

In her studies addressing work activity analysis through SEBE-based interviews, Le Bellu $(2011,2016)$ mentioned introspection without developing its contribution to the analysis but another research team (Rieken et al. 2015) recently published this kind of study and explicitly referred to introspection in the title of the paper. According to Rieken et al. (2015: 256), "Introspection in digital ethnography does not rely upon a mental representation of events but instead on shareable digital account of events" in that it "gives both the participating introspecting subject and the inquiring interviewer equal access to the first-person perspective digital representations".

It can be agreed that introspection in digital ethnography relies on shareable digital account of events but, before reaching the "subject-analyst" sharing level, another level, prior to this mentioned by Rieken et al., must be considered: the "subject-subject" level. Indeed, during the replay interview (as for any self-confrontation using video), the first one concerned by sharing events is the subject him/herself. This is why self-confrontation is called "self"- "confrontation". This consideration suggests that the first part of Rieken et al.'s sentence might be wrong as the subject is confronted with events experienced in the past for which the first-person perspective of the subfilm summons mental representations of what happened at this time.

In other words, the SEBE approach deconstructs Comte's argument saying that the subject cannot split in two (Comte 1830), because here, due to video self-confrontation, splitting the subject in two becomes effective. Indeed, if a lapse in time $\Delta t$ separates the realization of the activity happening at time $\mathrm{t}$ and the replay interview, thus happening at time $\mathrm{t}+\Delta \mathrm{t}$, the context of the replay interview brings together the conditions necessary for submitting the subject's Self at $\mathrm{t}+\Delta \mathrm{t}$ (noted Self $f_{t+\Delta t}$ ) watching and subjectively re-experiencing a past activity that might give him/her access to the re-enactment of event experienced by the Self at $t$ (noted Self ${ }_{t}$. Hence, provided that the conditions of introspection are effective during the replay interview, the subject may undertake introspection by the Self $f_{t+\Delta t}$ on the Self. As suggested by Clot (2001: 258) referring to Vygotsky, " 'being' does not coincide with the phenomenon (in other words, the 'real' with the 'realized') and even introspection does not abolish this difference. Because the mind is not only subject to it. It is divided into object and subject: my joy and my introspective understanding of this joy are different things" (pp. 273-274). Similarly, the Self $f_{t}$ s event and the Self $_{t+\Delta t}$ 's understanding of this event are two different things, because they address two different processes (occurring and understanding) and it involves two different Selfs (Self $f_{t}$ and $\left.\operatorname{Self}_{t+\Delta t}\right)$. This refers to the third-person perspective with the Self observing the Self (Libby and Eibach 2014) rather than to the objective state of mind proposed by Noa et al. (2018) in which the Self perceives him/herself from the imagined perspective of another person. This proposal of multiple Selfs was already envisaged by Overgaard (2006: 630): “ 'The Self' or the subject is obviously not identical to the content of his or her consciousness; for instance, the subject enjoys an uncountable number of conscious throughout his or her lifetime. Were the subject identical to conscious content, he or she would be as many selves as possible number of contents, continuously beginning and ceasing to exist."

All this leads to the assumption that introspection by the Self $f_{t+\Delta t}$ on the Self $f_{t}$ might be possible during the replay interview. This assumption matches Rieken et al. (2015) writings; for them, introspection in SEBE effectively occurs during the self-confrontation interview when the subjects view subjective (first-person) recording of their activity. So how was this done in Rieken et al.'s study? The main problem with this work is that authors claimed using introspection and emphasized its social dimension, but they did not provide neither experimental evidence of that nor analysis of this process. Therefore, the contribution of introspection in Rieken et al.'s work and consequently of its social dimension remained at the stage of assumption.

The fact that the subject may undertake introspection by the $\operatorname{Self}_{t+\Delta t}$ on the $\mathrm{Self}_{t}$ provided that conditions of introspection are effective during the replay interview is fundamental. According to the literature review characterizing what these conditions may be (see Sect. 2.1.2), it is clear that if introspection happens during the replay interview, it is impossible to take place all along the interview, since the interview is mainly a detailed explanation of states of mind, goals, intents and actions that goes beyond the mere description of the mental state. Introspection in replay interview might take place from time to time, it might even be said that it happens very infrequently and lasts very short length of time for each. Indeed, during the replay interview, the Self $f_{t+\Delta t}$ (the subject watching the sub-film) comments and explains most of the time what is doing the $\operatorname{Self}_{t}$ (the subject in action in the sub-film) relying mainly on episodic memory; this is neither introspection nor retrospection. If introspection happens, it is when the Self $f_{t+\Delta t}$ provides a comment on the Self $f_{t}$ s action while viewing the introspected event in the sub-film or immediately after viewing it, combined with the fact that this comment is short and gives information about a Self,'s mental state or cognitive process about which neither the Self $f_{t+\Delta t}$ nor the Self $f_{t}$ are aware of before making the comment.

This description additionally shows that, if it is introspection, it is nevertheless different from the forms proposed by the main streams (see Sect. 2.1.1) in the sense of the reviews of Overgaard (2006) and Danziger (2015): for these authors, introspection is an interaction by the Self on the $\operatorname{Self}_{t,}$ whereas here the interaction is by the Self $f_{t+\Delta t}$ 
on the Self $f_{t}$. Instead of introspection (that we should name direct introspection for reasons of accuracy), in this case we had retrospective introspection [or indirect introspection as proposed by Titchener (1912: 491)] which is triggered by self-confrontation and not opposite as claimed by others (see Rieken et al. 2015: 260). As recalled by Kriegel (2013: 1172), direct introspection occurs simultaneously with the introspected event (summoning short term memory), whereas indirect introspection occurs later and involves recollection of past events, relying on episodic memory or procedural memory (Piccinini 2003) (Fig. 1).

Lahlou's analyses of replay interviews in SEBE suggested that indirect introspection might be effective during replay interviews. He noticed that replay interviews could address events that could remain unconscious and could be remembered with accuracy: "as the human machine is designed for economy of attention, most of what we can do without cluttering our limited attention and consciousness span is done automatically (that is: often below the conscious threshold), and not stored in explicit memory" (Lahlou 2011: 620); "Probably because the recordings contain rich situated visual, auditory and kinetic cues which evoke re-enactment, participants recall with great detail their mental states at the time they acted, and can verbalize them, including their goals and sometimes sensations (e.g., thirst) [...] It seems that the more similar the context of memory retrieval is to the context of memory encoding, the better is the recall, and that having multimodal cues helps, especially when they are spatial or motor-see the enactment effect (Engelkamp and Cohen 1991). In other words, re-living the situation from first-person perspective would facilitate recalling one's own actions and mental states/processes" (Lahlou et al. 2015: 5). What is recalled is better anchored in memory due to the fact that what is recalled was experienced in action involving both mind and body (see for example Engelkamp and Zimmer 1989; Cohen 1989; Engelkamp and Cohen 1991; Engelkamp et al. 2005; Madan and Singhal 2012; Schult et al. 2014). These considerations led us to assume that a kind of indirect introspection might occur during SEBE interviews, a kind of long term indirect introspection involving shifted verbal reports not immediately consecutive to the introspected event but delayed by several hours: $\Delta t$ would be several hours instead of several seconds; this might be called introspection provided that the introspected mental state could be recalled at the moment of the introspective act from a non-conscious memory.

\section{Materials and methods}

The model mobilized for introspection during the SEBE replay interview was this of an interaction of the Self $t_{t+\Delta t}$ on the Self, relying on episodic memory. $\Delta \mathrm{t}$ is the lapse in time separating the realization of the activity and the replay interview. This assumed that introspection should occur in its indirect form.

\subsection{Design}

The replay interview of a case study regarding the application of a SEBE method was considered. The SEBE method was applied to analyze activities at a French nuclear power plant during operating situations (results regarding the work activity analysis were already published (Fauquet-Alekhine and Daviet 2015) and performed by Operations shift teams as part of their routine work. The activity chosen for the study was performed by a nuclear reactor pilot in the control room, common to any pilots' work: in French "le tour de bloc" which may be translated as "block watch-around". This consists in watching and checking operating parameters in the control room. This activity lasts usually from 10 to $30 \mathrm{~min}$. The SEBE-based analysis of this activity was undertaken in the framework of an overall improvement of workers' competencies at Chinon nuclear power plant (France) and the activity was chosen for investigation addressing "introspection", because it does not last long and does not imply complex technical gestures; the consecutive interview is, therefore, easy too. The protocol of the SEBE method is described in Sect. 3.3. Among the cases analyzed in the framework of an overall improvement of workers' competencies, one was chosen as a case study (Sect. 3.5) to undertake an analysis of how "introspection" could happen during the interview of self-confrontation in SEBE. Due to the heaviness of the analysis, it was limited to one case study, i.e., one subject and one occurrence of introspection. The protocol of the analysis for introspection during the replay interview is described in Sect. 3.4.

\subsection{Apparatus for the SEBE method applied to the case study}

The digital ethnography equipment was made up of three parts linked with cables: (1) a micro audio digital recorder DVR-500-HD2 self-powered by internal batteries, not much bigger than a mobile phone, (2) a $4 \mathrm{~mm}$ diameter, $40 \mathrm{~mm}$ length miniaturized camera (subcam: Lahlou 1999) mounted on safety glasses, (3) a lavaliere microphone. This SEBE equipment was assembled from components produced at Active Media Concept (website: www.amc-tec.com). This equipment fulfilled the requirements of video quality, energy 
autonomy, data storage, size and industrial environment disturbance. The main advantage of this equipment was to be adaptable to any kind of glasses (safety or vision).

\subsection{Protocol for the SEBE method applied to the case study}

The procedure applied for the SEBE-based work activity analysis was developed, tested and validated in a previous work (Fauquet-Alekhine 2016a) and was applied in other studies (Fauquet-Alekhine and Lahlou 2017a, b; FauquetAlekhine et al. 2018).

The schema of the protocol was the following:

- At the beginning of the shift, the analyst exchanged with the Operations team during the shift briefing to identify who would be volunteer to be involved in the activity analysis.

- Workers were informed of the aim of the study, signed an informed consent and undertook a risk assessment regarding the subcam used in real operating situation (Fauquet-Alekhine 2016b; Fauquet-Alekhine et al. 2018). Then they were equipped with the subcam. This lasted about $5 \mathrm{~min}$. for each subject (preparation phase).

- Workers performed their activity (lasting from 10 to 30 min.), recording at the same time a subjective video (subfilm) of their activity (capture phase). During this phase, no verbalization (speaking out loud) was requested but this was not forbidden if the worker was used to doing so.

- At the end of the activity, workers gave the subcam equipment back to the analyst and returned to their work; during this time, the analyst performed a preanalysis of the subjective video for the workers to select video sequences of interest and prepare possible questions.

- After the activity or during the following shifts, subjects were met individually to undertake the replay interview (analysis phase). The replay interview was based on self-confrontation and explicitation techniques. A post-analysis of the interviews (recorded) was carried out by the analyst to produce the output data regarding improvement of workers' competencies (Fauquet-Alekhine and Daviet 2015).

- These resulting output data were finally discussed with representatives of the professions for validation (validation phase).

As mentioned in Sect. 1, the replay interview took from techniques of self-confrontation and explicitation interview described hereinafter. This method is similar to the cued recall debrief developed by Omodei and McLennan
(1994) and applied by others (see for example Bentley et al. 2005; Rix and Biache 2004). In addition, during the replay interview, the work activity analysis was based on the Square of PErcieved ACtion model (SPEAC model) proposed by Fauquet-Alekhine (2016a). The SPEAC model describes the necessary conditions for the subject to successfully put competencies in action and helps the analyst to question the worker regarding the motives of the activity, the professional experience, the means and the motivation. This thus helped the analyst to question the workers during the replay interview.

\subsection{Protocol of the analysis for introspection during the replay interview}

The SEBE replay interview was video recorded from a thirdperson perspective. It was then carefully analyzed to identify possible occurrences of introspection. The criteria to select moments of possible introspection were these defined from the literature background, the structure of these moments being compared with the characteristics of introspection provided by the characterization of introspection during SEBE interviews (see Sects. 2.1.2 to 2.1.4). A refined description of such a moment was used to understand the introspection process. Due to the heaviness of the analysis, only one occurrence of introspection was examined.

\subsection{Subjects}

Subjects' participation to the SEBE-based work activity analysis was voluntary $(N=10)$. They filled in a form regarding individual data: gender, age and professional experience. Age was asked per range; for example, range 21-30 years, range $31-40$ years and so on. Professional experience was asked in years according to the time spent in the position. Table 1 presents the average characteristics of the sample of subjects participating in the SEBE-based work activity analysis.

For analysis of the introspection, the selection of a subject was based on the least squares calculated using individual data and the mean values in Table 1. The subject (reference subject: \#OpJ) selected as a study case for introspection was male, presented the professional experience (5 years) closer to the mean value (4.8 years) and was in the second

Table 1 Subjects' characteristics involved in the studies activities

\begin{tabular}{ll}
\hline Characteristics & $\mathrm{M}(\mathrm{SD})$ \\
\hline Gender (\% male) & 90 \\
Age (range) & Range 3: \\
& $31-40$ years \\
Experience (years) & $4.8(4.9)$ \\
\hline
\end{tabular}


range of age (21-30 years); the selected subject presented the minimum value for least squares thus corresponding to the minimal distance from the mean values of gender, age and experience.

\subsection{Ethics}

All participants signed an informed consent before undertaking experiments. This study received ethical approval (Code of Approval: DSP/RS/PFA-4) of the Ethics Committee of the Dept. of Social Psychology (LSE, UK) and has, therefore, been performed in accordance with the ethical standards laid down in the 1964 Declaration of Helsinki. The person selected in the case study has agreed to waive anonymity and appear in clear on the photographs. The person signed an informed agreement to this effect.

\section{Results}

We considered the replay interview for the activity "block watch-around" undertaken with a subject (reference \#OpS, experiment TEST-IND-OP-01) selected as a representative of the workers sample as explained in Sect. 3.5. The video recording of the replay interview was carefully analyzed in the light of the model proposed to depict introspection in Sect. 3.4.

During the replay interview which was video recorded, to depict the way he was checking monitors while performing the activity (excerpt of the subjective video in Fig. 2), the

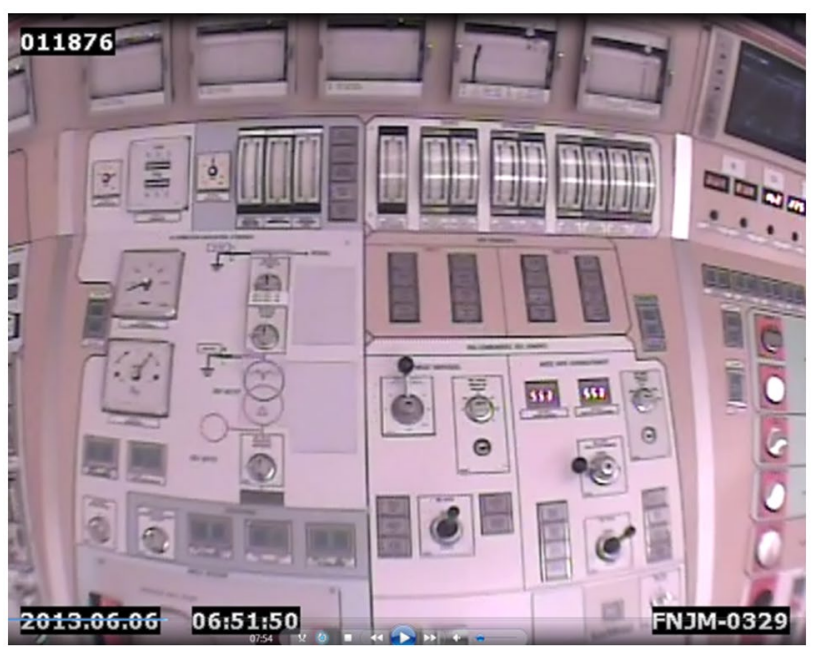

Fig. 2 Excerpt of the pilot's subfilm, while the video field remained unchanged for a few hundreds of milliseconds during the activity "bloc watch-around". In the upper part of the picture, five paper monitors provide prints of physical parameters with time. Source: data subcam et al.Isimu pil MS1 and MSI-062013 20130606 part1 (Op J)—file $1 \mathrm{Go}-\mathrm{t}=07^{\prime} 54^{\prime \prime}$ pilot said "je regarde si ça tire droit" (I watch to see whether it goes straight). Consecutive exchanges between the pilot and the analyst showed that this meant the pilot did not read the values of parameters on the monitors: it was easier and faster to check a signal position rather than read the value according to the scale of the monitors and compare it with the expected values. He said this was done without losing any reliability on values. The analysis for introspection during the replay interview allowed us to assuming an introspection occurrence when detecting this tacit knowledge during the subject-analyst interaction (illustrated in Fig. 3).

The sequences of the replay interview are described in the following (Reference File: interview replay OpJ 20,130,821-file 1 Go):

- At $\mathrm{t}_{\mathrm{r}}=18^{\prime} 09^{\prime \prime}$ : Identification of specific moment for the Self.

Note: $\mathrm{t}_{\mathrm{r}}$ is the time associated with the replay interview recording.

While the subject (the $\operatorname{Self}_{t+\Delta t}$ ) and the analyst were together watching the activity video during the replay interview, the subject acting in the subfilm (the $S e l f_{t}$ ) stopped in front of monitors on the control panel (see excerpt of the subfilm in Fig. 2: the video field remained unchanged for a few hundreds of milliseconds), suggesting that the subject was looking at or thinking about something. The analyst asked what was going on $\left(\mathrm{t}_{\mathrm{r}}=18^{\prime} 09^{\prime \prime}\right)$ and the subject, who had his right hand on the mouse, stopped the video player $\left(\mathrm{t}_{\mathrm{r}}=18^{\prime} 09^{\prime \prime}\right)$.

- At $\mathrm{t}_{\mathrm{r}}=18^{\prime} 10^{\prime \prime}$ :

The subject said "je regarde si ça tire droit sur les enregistreurs" (I look to see whether it goes straight on the recording monitors). This was verbal reports by the Self $f_{t+\Delta t}$ about the $\operatorname{Self}_{t}$ to qualify the specific moment (from $\mathrm{t}_{\mathrm{r}}=18^{\prime} 10^{\prime \prime}$ to $18^{\prime} 12^{\prime \prime}$.

- At $\mathrm{t}_{\mathrm{r}}=18^{\prime} 12^{\prime \prime}$ :

Continuing the first comment, the subject spontaneously gave details about the physical quantities monitored. This was verbal reports by the $\operatorname{Self}_{t+\Delta t}$ about the $\operatorname{Self}_{t}$ to spontaneously explain the specific moment (from $\mathrm{t}_{\mathrm{r}}=18^{\prime} 12^{\prime \prime}$ until $\left.\mathrm{t}_{\mathrm{r}}=18^{\prime} 14^{\prime \prime}\right)$.

- At $\mathrm{t}_{\mathrm{r}}=18^{\prime} 26^{\prime \prime}$ :

The analyst took notes and then asked $\left(t_{\mathrm{r}}=18^{\prime} 26^{\prime \prime}\right)$ what he meant by the expression "goes straight". The pilot explained $\left(\mathrm{t}_{\mathrm{r}}=18^{\prime} 34^{\prime \prime}\right.$ until 18'54") the goal of this way of working: he did not read the values of parameters for 
(a)

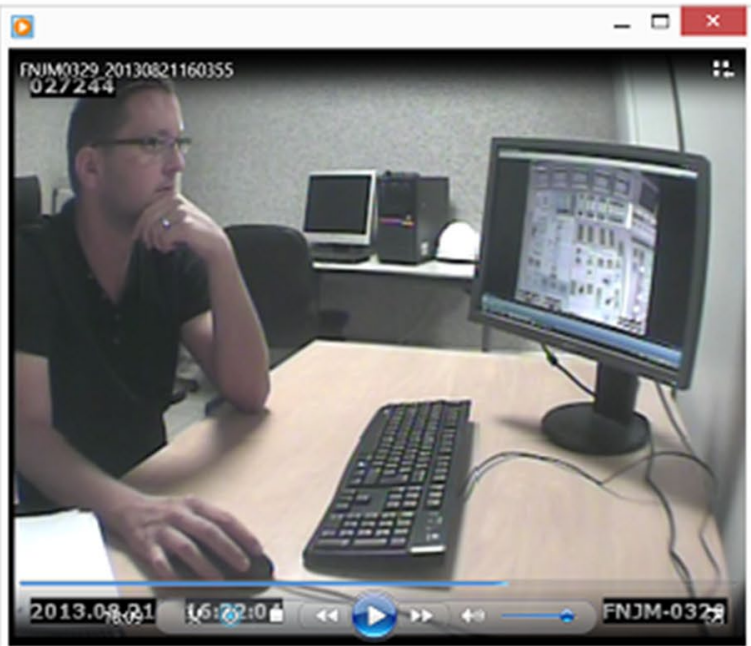

(c)

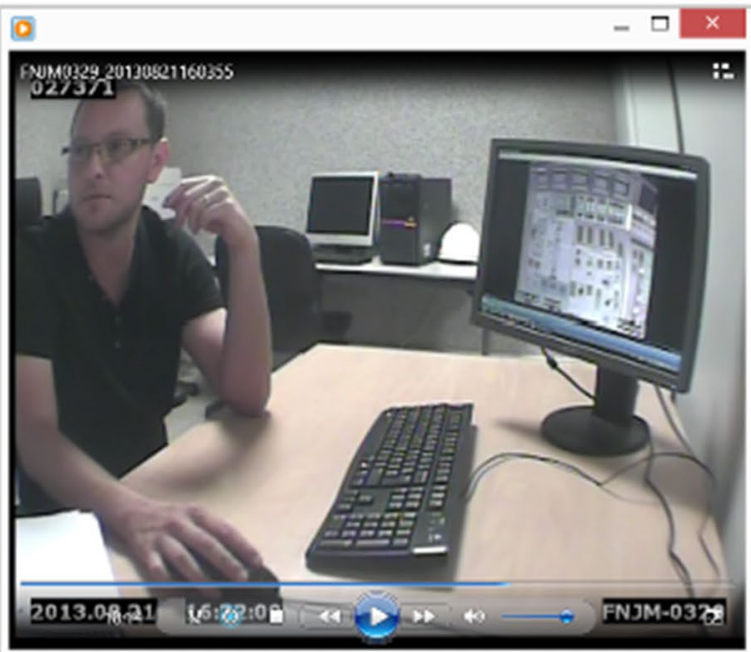

Fig. 3 Sequences of replay interview including introspection. a $\mathrm{t}_{\mathrm{r}}=18^{\prime} 09^{\prime \prime}$ - the subject stopped the video player showing the specific moment (subject's mouth is closed). $\mathbf{b} \mathrm{t}_{\mathrm{r}}=18^{\prime} 11^{\prime \prime}$ - the subject qualified the specific moment (subject's mouth is opened). $\mathrm{ct}_{\mathrm{r}}=18^{\prime} 14^{\prime \prime}-$

certain monitors or indicators; it was easier and faster to check a signal position rather than read the value according to the scale of the monitor and compare it with the expected value. Further questions and answers (after $\mathrm{t}_{\mathrm{r}}=18^{\prime} 54^{\prime \prime}$ until $\left.20^{\prime} 14^{\prime \prime}\right)$ showed that this was done without losing any reliability on values. When he was asked whether this practice was his own, the subject said that most of his colleagues (even all) did so. When he was asked, where he was taught this practice, he could not find any answer. This was verbal reports by the $\operatorname{Self}_{t+\Delta t}$ about the Self $f_{t}$ to explain the specific moment (from $\mathrm{t}_{\mathrm{r}}=18^{\prime} 26^{\prime \prime}$ until $\mathrm{t}_{\mathrm{r}}=20^{\prime} 14^{\prime \prime}$ ) when answering questions. During this sequence, verbal reports were not spontaneous but induced by questions, conversely to previous sequences. (b)

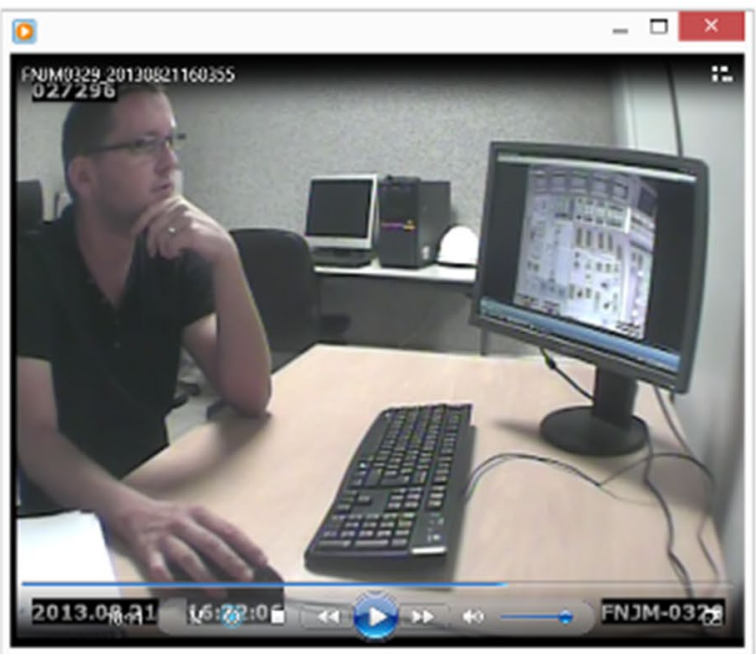

(d)

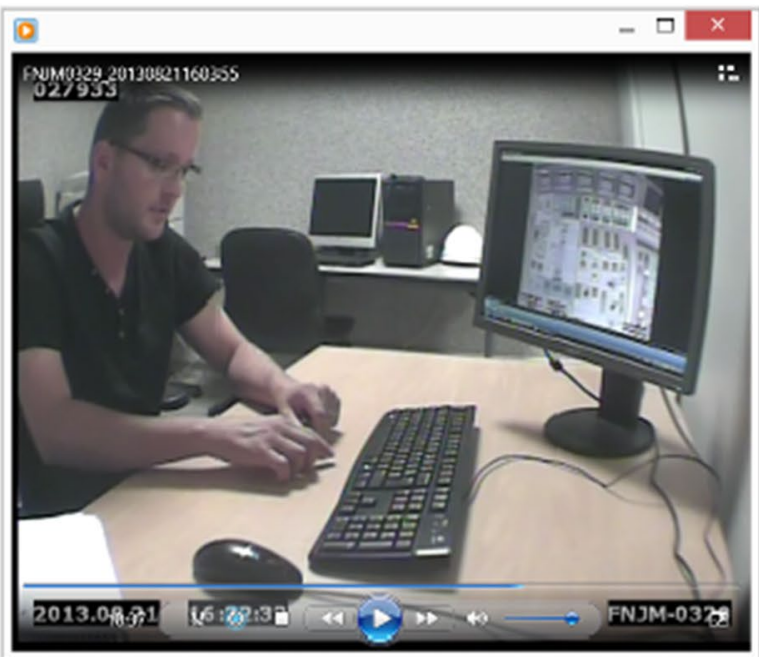

the subject spontaneously gave details about the specific moment. d $\mathrm{t}_{\mathrm{r}}=18^{\prime} 37^{\prime \prime}$ - the subject explained the specific moment (with gesture). Note: $\mathrm{t}$ is the time associated with the replay interview recording

This description shows that it followed a process made up of two distinct steps:

- A focus of the subject Self $f_{t+\Delta t}$ on a given mental state triggered by the analyst corresponding to the step "Identification of specific moment for the Self." at $\mathrm{t}_{\mathrm{r}}=18^{\prime} 09 "$.

- A self-description of the mental state by the subject Self $t_{t+\Delta t}$ through an immediate, consecutive brief verbal report corresponding to the step "Verbal reports from the Self $t_{t+\Delta t}$ about the Self to qualify the specific moment" from $\mathrm{t}_{\mathrm{r}}=18^{\prime} 10^{\prime \prime}$ to $18^{\prime} 12^{\prime \prime}$.

This two-time segment (identifying and qualifying) fulfils Gaillard's criteria described in section. 


\subsection{Necessary for introspection}

- Instructions given to subject for verbalization were short enough ("what was going on ?") to minimize the effect on the cognitive process of introspection: the verbal report was neither limited to a few possible categories such as "yes" or "no" nor forced to be an elaborated and exhaustive description or the introspected event.

- Despite its metaphorical form, the verbal report was informative enough to be considered as complete regarding the subject's own cognitive processes. It was verbalized in a simple form and thus minimized the disturbance of the current cognitive process in progress.

- The verbal report was consistent with the third-person observation and analysis of the video undertaken by the analyst.

These elements match the model proposed to describe introspection during SEBE interview; an indirect introspection of the Selft $+\Delta t$ on the Selft, where $\Delta t$ is the lapse in time between the realization of the activity and the replay interview.

However, the indirect introspection was shifted in time by several hours when compared with the task carried out (average delay (in hours) for subjects involved in the SEBE-based work activity analysis was $M=59.1$ with $\mathrm{SD}=39.6)$. When comparing the time related to the action (performing the block watch-around) and the time related to the occurrence of introspection, it is obvious that this did not comply with the maximum $5 \mathrm{~s}$-criterion suggested by Ericsson for verbal reports (see Sect. 2.1.2 and Ericsson 2003). Measuring the time criterion this way means that the action relates to the Selft $+\Delta t$, while the occurrence of introspection relates to the Selft. The fact that the two points relate to different Selves need to be discussed.

Regarding the memory aspects, episodic memory is obviously called upon during the phase of verbal report to qualify the specific moment. Identifying the implication of other forms of memory should be discussed.

\section{Discussion}

\subsection{Process of introspection}

Section 4.1 characterized how indirect introspection could occur during a SEBE replay interview. Results obtained from the case study showed that indirect introspection occurred according to a two phase segment (identifying and qualifying):

- "Identification of specific moment for Self" at $\mathrm{t}_{\mathrm{r}}=18^{\prime} 09 "$ ".
At this time of the replay interview, there was a focus of the subject $S_{e l f} f_{t+\Delta t}$ on a given mental state triggered by the analyst. This corresponded to the first introspective phase: the identification of the mental state. This was triggered by the analyst's question, because the action going on at this moment on the subfilm was just a usual action in the subject's opinion. This is why the subject neither stopped the video by himself nor made any spontaneous comment. While watching the video, the subject probably re-enacted the activity due to "situated visual, auditory and kinetic cues" contributing to "recall with great detail their mental states at the time they acted" as suggested by Lahlou et al. (2015: 220). We may suggest here two possibilities regarding the cognitive process underpinning this identification: (1) the re-enactment effect recreated the same unconscious mental state for the subject while viewing the sub-film and the mind was made conscious at the moment of the analysts' question, (2) the analysts' question summoned the subject's implicit memory storing previous experiences to carry out a task without conscious awareness of this (see Fig. 1), i.e., the procedural memory. Whatever the possibility explaining the process, it is highly likely that the mental state identified was unconscious for the subject before identification.

- "Verbal reports from the Self $f_{t+\Delta t}$ about the Self" to qualify the time segment $t_{r}=18^{\prime} 10^{\prime \prime}$ to $18^{\prime} 12^{\prime \prime}$.

At this time of the replay interview, there was a second introspective phase: the self-description of the mental state by the subject $\operatorname{Self}_{t+\Delta t}$.

The subject said "je regarde si ça tire droit sur les enregistreurs" (I watch to see whether it goes straight on the recording monitors). The metaphorical expression used for this immediate consecutive verbal report was natural, simple, and contained all the information necessary to depict the mental state, thus minimizing the disturbance of the current cognitive process (Titchener 1912; Ericsson 2003; Danziger 2015). The problem here was that all the information necessary to depict the mental state was concentrated and hidden within this simple metaphorical verbal report.

This two-time segment was then complemented by an explanation phase:

- Verbal reports from the $\operatorname{Self}_{t+\Delta t}$ about the Self for explanation after $\mathrm{t}_{\mathrm{r}}=18^{\prime} 12$ ".

At this point, the indirect introspection was completed and the subject entered a post-introspective phase: the introspected mental state had become conscious and the subject was definitely engaged in a cognitive process of description. This phase relied on procedural memory to describe the metaphorical introspective verbal report and relied on episodic memory when the subject gave details regarding 
the parameters checked, the context of checking and the subject's goals (Fig. 1).

This kind of long term indirect introspection might have happened several times per interview. They were so short (in the case study, the two-time segment did not last more than 3 s.) that they have gone unnoticed.

\subsection{Characteristics of introspection}

Given this, it is interesting to compare what was observed during the episode indirect introspection in the case study to the key points of the literature background (Sect. 2).

The necessity to implement strict and laboriously trained methodological procedures in the aim to access introspection is not verified for the subject: in the study case, the subject was a passive interviewee. The finding is less clear for the analyst: although not trained to investigate introspection, the analyst was an interviewer trained to apply self-confrontation with explicitation and a SPEAC-based questioning; this has nothing to do directly with introspection; however, this might have been a set a favoring factors.

The opposite effect of simple vs elaborate questions was verified: in the case study, a simple question led to a simple and short answer that gave rise to the occurrence of introspection (the two-time segment) and the following elaborate questions triggered an explanation phase, the subject being engaged in a cognitive process of description.

Finally, the time lapse suggested by Ericsson was verified provided that the correct elements are considered: as demonstrated in Sect. 2.1.4, what must be compared in SEBE interviews is not the time related to the action vs the time related to the occurrence of introspection; this would result in comparing action and introspection from the only perspective of the Self. Doing so, action and occurrence of introspection are related to the same Self. What must be considered is the action from the perspective of the Self and the introspection from the perspective of the Self $t_{t+\Delta t}$. This lapse of time was estimated at $3 \mathrm{~s}$, value of the same magnitude of this of Ericsson (about $5 \mathrm{~s}$ ).

\subsection{Discussion regarding benefits of introspection for SEBE}

The fact that introspection occurs one or several times during a SEBE-based interview is interesting in terms of process. Provided that the analyst is able to seize such a moment (even when not being aware that introspection is in progress) and to obtain clarifications about what is described during the activity and what is felt by the subject, the moment of introspection is a benefit for the work activity analysis, because it focuses the analyst on a point of interest during the activity. This process may go unnoticed: it works unbeknownst to the analyst and the subject as demonstrated by the study case.

Similarly, identifying precisely moments of introspection and undertaking an in-depth analysis of the related process does not yet present any added-value for the SEBE-based interview during work activity analysis. This is illustrated when comparing the results presented in Sect. 4.2 and these of the SEBE-based work analysis in Fauquet-Alekhine and Daviet (2015): detecting introspection did neither change the results nor the conclusions of the study.

\subsection{Metaphors}

In addition, the study case associated a metaphorical expression with the introspection occurrence. We even argue that this metaphorical expression indicated that introspection was actual: the subject's verbal report was informative enough to be considered as complete and was verbalized simply and thus minimized the disturbance of the current cognitive process. Perhaps this would not have been possible with a literal expression. This remark does not mean that all introspections are metaphorical or that, every time a metaphor is noted in the speech, introspection occurs.

As the language used by subjects to describe their work activity is not innocuous, the results of the case study show that metaphors may be a relevant source for understanding the activity beyond the literal meaning of the words or sentences. Psycholinguistic studies have been undertaken regarding discourse analysis and communication in the workplace (see for example: Roth 2004; Andrén et al. 2010; Fauquet-Alekhine 2010, 2017a, b) and have contributed to the demonstration that language through the discourse or the narrative may carry implicit depictions of the subjects' activities. Among these implicit elements of language, metaphors may offer new angles of comprehension of the subjects' activity (Glucksberg 2003; Steen 2011; Veraksa 2013). Therefore, the detection and analysis of metaphorical language as a tool for work analysis during the replay interview is an aspect which should be studied in more depth.

\section{Conclusions}

We reviewed controversies over introspection and followed the Brentano middle way between a Positivist (Comte, Mills et al.) categorical denial of validity and Wundtian dead ends of methodology to isolate 'pure sensation' with the right attitude. The key question was not whether introspection is valid, but under which circumstances. This posed the need for supporting methodology such as video replay and selfconfrontation interviewing (e.g., SEBE method) which elicit 
verbal reports that are eminently informative for the analysis, understanding and improvement of work and other everyday activities.

The notion of introspection (direct and indirect) was clarified when occurring during a SEBE interview and criteria specifying circumstances of introspection occurrence were given. For the first time, an in-depth analysis of introspection during replay interviews was undertaken. It was found that introspection could occur during the SEBE-based interview and could remain unnoticed. Also, the form of introspection was found indirect $\left(\right.$ Self $_{t+\Delta t} /$ Self $_{t}$ ) and the process of introspection was described as a two-time segment (identifying and qualifying) lasting a few seconds.

In the case study, we found that being aware of the occurrence of introspection did not give any added-value to the SEBE analysis; however, this occurrence, even being unnoticed, was an actual benefit by giving access to a nonexplicit aspect of the work activity analyzed. Furthermore, metaphorical characters of the subjects' speech could be a relevant indicator for introspection detection, easier to detect than introspection.

The association metaphor/introspection occurrence seems important: in the case study, introspection might have not happened without the metaphor. This remark highlights two points: (1) might introspection occurrence be always related to a metaphorical expression during a SEBE-based interview? (2) considering the fact that it helps the analysts to identify a tacit know-how, might introspection and/or metaphor be an indicator of a possible tacit knowledge or know-how? Both needs further experiments.

Acknowledgements The authors thank all participants from the Nuclear Power Plant and the Training Center of Chinon (France) for their contribution. The authors thank especially Julien Castellarin, nuclear reactor pilot, who accepted to illustrate this paper with his image. The authors thank Active Media Concept (www.amc-tec.com) for efficient technical support. Research was financially supported by Electricité de France.

\section{Compliance with ethical standards}

Conflict of interest Philippe Fauquet-Alekhine is Scientific Director INTRA Robotics lab (a branch of Electricité de France) and is former Human Factors Consultant, expert in Innovative Development for the Operational Professionalization at Chinon Nuclear Power Plant, Electricité de France (France).

Open Access This article is licensed under a Creative Commons Attribution 4.0 International License, which permits use, sharing, adaptation, distribution and reproduction in any medium or format, as long as you give appropriate credit to the original author(s) and the source, provide a link to the Creative Commons licence, and indicate if changes were made. The images or other third party material in this article are included in the article's Creative Commons licence, unless indicated otherwise in a credit line to the material. If material is not included in the article's Creative Commons licence and your intended use is not permitted by statutory regulation or exceeds the permitted use, you will need to obtain permission directly from the copyright holder. To view a copy of this licence, visit http://creativecommons.org/licenses/by/4.0/.

\section{References}

Andrén M, Sanne JM, Linel P (2010) Striking the balance between formality and informality insafety-critical communication: train traffic control calls. J Pragmat 42:220-241

Atkinson R, Shiffrin R (1968) Human memory: a proposed system and its control processes. In: Spence KWSJ (ed) The psychology of learning and motivation. Academic Press, New York, pp 89-195

Bechara A, Damasio H, Tranel D, Damasio AR (1997) Deciding advantageously before knowing the advantageous strategy. Science 275:11293-11295

Bentley T, Johnston L, Baggo, KV (2005) Evaluation using cuedrecall debrief to elicit information about a user's affective experiences. In: Citizens online: considerations for today and the future. OZCHI '05 Proceedings of the 17th Australia conference on Computer-Human Interaction for the Computer-Human Interaction Special Interest Group (CHISIG) of Australia Narrabundah, Australia

Brentano F (1874) Psychologie vom Empirischen Standpunkte, Leipzig, Verlag von Duncker and Humblot

Clot Y (2001) Clinique du travail et action sur soi. In: Baudouin JM, Friedrich J (eds) Théories de l'action et education. Louvain-laNeuve, De Boeck Supérieur, pp 255-277

Cohen RL (1989) Memory for action events: the power of enactment. Educ Psychol Rev 1(1):57-80

Comte, A. (1830/1990). Cours de philosophie positive. Paris: Hermann

Danziger K (2015) Introspection: history of the concept. Int Encyclop Soc Behav Sci 12:702-704

Engelkamp J, Cohen RL (1991) Current issues in memory of action events. Psychol Res 53(3):175-182

Engelkamp J, Zimmer HD (1989) Memory for action events: a new field of research

Engelkamp J, Seiler KH, Zimmer HD (2005) Differential relational encoding of categorical information in memory for action events. Memory Cognit 33(3):371-379

Ericsson A (2003) Valid and non-reactive verbalization of thoughts during performance of tasks: towards a solution to the central problems of introspection as a source of scientific data. J Conscious Stud 10(9-10):1-18

Ericsson KA, Simon HA (1980) Verbal reports as data. Psychol Rev $87: 215-251$

Fauquet-Alekhine PH (2010) Надежность рабочего сообщения для операторов ядерных реакторов: изучение на тренажерах, анализ случаев и укрепление безопасности [Reliability of operational communication for pilots of nuclear reactors: studies on simulators, events analysis, and reinforcement of safety]. In: Morozova L, Weider (eds) Prosodie, traduction, fonctions [Prosody, translation, functions]. EME and Intercommunications, Brussels, pp 207-210

Fauquet-Alekhine PH (2016a) Subjective ethnographic protocol for work activity analysis and occupational training improvement. $\mathrm{Br}$ J Appl Sci Technol 12(5):1-16 (BJAST.21632)

Fauquet-Alekhine PH (2016b) Risk assessment for subjective evidencebased ethnography applied in high risk environment. Adv Rese 6(2):1-13 (AIR.21597)

Fauquet-Alekhine PH (2017a) Psycholinguistic (analysis of operational communication part I): the standard usage of communication in high risk industries. Linguist J 12(1):86-109

Fauquet-Alekhine PH (2017b) Psycholinguistic analysis of operational communication (part II): prescribed communication for reliability in high risk industries. Eur J Lang Stud 4(2):27-40 
Fauquet-Alekhine PH, Daviet Fr (2015) Detection and characterization of tacit occupational knowledge through speech and behavior analysis. Int J Innov Manage Technol 6(1):21-25

Fauquet-Alekhine PH, Lahlou S (2017) The Square of PErcieved ACtion model (SPEAC model) applied in digital ethnography for work activity analysis: performance and workers' perception. Current J Appl Sci Technol 22(3):1-13 (CJAST.34985)

Fauquet-Alekhine PH, Le Bellu S, Buchet M, Berton J, Bouhours G, Daviet FR, Granry JC, Lahlou S (2018) Risk assessment for subjective evidence-based ethnography applied in high risk environment: improved protocol. Adv Res 16(3):1-15 (AIR.43259)

Gaillard V, Vandenberghe M, Destrebecqz A, Cleeremans A (2006) First-and third-person approaches in implicit learning research. Conscious Cogn 15(4):709-722

Glucksberg S (2003) The psycholinguistics of metaphor. Trends Cognit Sci 7(2):92-96

Goldman R, Pea R, Barron B, Derry S (2007) Video research in the learning sciences. Mahwah, NJ, Lawrence Erlbaum Associates

Knoblauch H, Schnettler B, Raab J, Soeffner HG (2006) Video-analysis methodology and methods: qualitative audiovisual data analysis in sociology. Frankfurt am Main: Berlin: Bern: Bruxelles: New York: Oxford: Wien, Peter Lang

Koehler W (1947) Gestalt Psychology. Meridian Books, NY

Kriegel U (2013) A hesitant defense of introspection. Philos Stud 165(3):1165-1176

Lahlou S (1999) Observing Cognitive Work in Offices. In N. Streitz, J. Siegel V. Hartkopf, S. Konomi (Eds), Cooperative buildings. Integrating information, organizations and architecture, Heidelberg, Springer, Lecture Notes in Computer Science (1670: 150-163)

Lahlou S (2011) How we can capture the subject's perspective? An evidence-based approach for the social scientist. Soc Sci Informat 50(3-4):607-655

Lahlou S, Le Bellu S, Boesen-Mariani S (2015) Subjective evidence based ethnography: method and applications. Integrat Psychol Behav Sci 49(2):216-238

Le Bellu S (2011) Capitalisation des savoir-faire et des gestes professionnels dans le milieu industriel. PhD thesis, Un. of Bordeau, 2011BOR21825

Le Bellu S (2016) Learning the secrets of the craft through the realtime experience of experts: capturing and transferring experts' tacit knowledge to novices. PISTES, 18-1. http://pistes.revues. org $/ 4685$

Libby LK, Valenti G, Hines KA, Eibach RP (2014) Using imagery perspective to access two distinct forms of self-knowledge: associative evaluations versus propositional self-beliefs. J Exp Psychol Gen 143(2):492-497

Madan CR, Singhal A (2012) Using actions to enhance memory: effects of enactment, gestures, and exercise on human memory. Front Psychol 3:507

Maia TV, McClelland JL (2004) A reexamination of the evidence for the somatic marker hypothesis: what participants really know in the Iowa gambling task. Proc Natl Acad Sci USA 101(45): 16075-16080

Nisbett RE, Wilson TD (1977) Telling more than we can do: verbal reports on mental processes. Psychol Rev 84:231-259

Noah T, Schul Y, Mayo R (2018) Thinking of oneself as an object of observation reduces reliance on metacognitive information. J Exp Psychol Gen 147(7):1023-1042

Omodei M, McLennan J (1994) Studying complex decision making in natural settings: using a head mounted video camera to study competitive orienteering. Percept Mot Skills 79:1411-1425

Omodei MM, McLennan J, Wearing JW (2005) How expertise is applied in real-world dynamic environments: head-mounted video and cued recall as a methodology for studying routines of decision making. In: Betsch $\mathrm{T}$, Haberstroh $\mathrm{S}$ (eds) The routines of decision making. Lawrence Erlbaum Associates, Mahwah, New Jersey, pp 271-288

Overgaard M (2006) Introspection in science. Conscious Cogn 15:629-633

Pea RD (1994) Seeing what we build together: distributed multimedia learning environments for transformative communications. J Learn Sci 3(3):285-299

Petitmengin C (2009) The validity of first-person descriptions as authenticity and coherence. J Conscious Stud 16(10-12):252-284

Piccinini G (2003) Data from introspective reports: upgrading from common sense to science. J Conscious Stud 10(9-10):141-156

Rieken J, Garcia-Sanchez E, Trujillo MP, Bear D (2015) Digital ethnography and the social dimension of introspection: an empirical study in two Colombian schools. Integrat Psychol Behav Sci 49(2):253-274

Rix G, Biache M (2004) Enregistrement en perspective subjective située et entretien en re situ subjectif: une méthodologie de constitution de l'expérience. Intellectica 1(38):363-396

Rix-Lièvre G, Lièvre P (2010) An innovative observatory of polar expedition projects: an investigation of organizing. Project Manage $\mathrm{J}$ 41(3):91-98

Roth WM (2004) Perceptual gestalts in workplace communication. J Pragmat 36:1037-1069

Schult J, Von Stülpnagel R, Steffens MC (2014) Enactment versus observation: Item-specific and relational processing in goaldirected action sequences (and lists of single actions). PLoS ONE 9(6): 99985

Schwitzgebl E (2004) Introspective training apprehensively defended. J Conscious Stud 11(7-8):58-76

Steen GJ (2011) The contemporary theory of metaphor-now new and improved! Rev Cognit Linguist 9(1):26-64

Theureau J (2002) L'entretien d'autoconfrontation comme composante d'un programme de recherche empirique and technologique. Paper presented at the IIème Journées Internationales des Sciences du Sport, Paris. 12-15 Nov 2002

Titchener EB (1912) The schema of introspection. Am J Psychol 23:485-508

Tofel-Grehl C, Feldon DF (2013) Cognitive task analysis-based training: a meta-analysis of studies. J Cognit Eng Dec Making 7(3):293-304

Velez-Pardo C, Jimenez-Del-Rio M (2015) From dark to bright to gray sides of memory: in search of its molecular basis and Alzheimer's Disease. Aus J Clin Neurol 2:5-1045

Veraksa AN (2013) Symbol as a cognitive tool. Psychology in Russia: State of the Art 1:57-65

Vermersch P (1994/2006) L'entretien d'explicitation. Issy-les-Moulineaux , ESF Editeur

Von Cranach M, Kalbermatten U, Indermühle K, Gugler B (1982) Goal-directed action. Academic Press, London

Weger U, Wagemann J, Meyer A (2018) Introspection in psychology. Eur Psychol 23(3):206-216

Wei J, Salvendy G (2004) The cognitive task analysis methods for job and task design: review and reappraisal. Behav Informat Technol 23(4):273-299

Publisher's Note Springer Nature remains neutral with regard to jurisdictional claims in published maps and institutional affiliations. 